\title{
Effect of Cu Nanoparticles on the Properties of PP-g-PHMG/PE Monofilament and Its Antifouling Application
}

\author{
Yue Wang $\mathbb{D}^{1,2}$ Feng Han, ${ }^{1}$ Wenwen Yu ${ }^{1},{ }^{3}$ Jiangao Shi $\mathbb{D}^{1},{ }^{1}$ Aiyan Shu, ${ }^{1,2}$ and Min $\mathrm{Zhang}^{2}$ \\ ${ }^{1}$ East China Sea Fisheries Research Institute, Chinese Academy of Fishery Sciences, Shanghai 200090, China \\ ${ }^{2}$ Shanghai Ocean University, Shanghai 201306, China \\ ${ }^{3}$ College of Materials Science and Engineering, Taiyuan University of Technology, Taiyuan 030024, China
}

Correspondence should be addressed to Wenwen Yu; yuwenwen666@163.com and Jiangao Shi; jiangaoshi666@163.com

Received 6 April 2021; Accepted 23 June 2021; Published 8 July 2021

Academic Editor: Huining Xiao

Copyright () 2021 Yue Wang et al. This is an open access article distributed under the Creative Commons Attribution License, which permits unrestricted use, distribution, and reproduction in any medium, provided the original work is properly cited.

The modified polyethylene monofilament was prepared by melt blending and spinning using polyethylene (PE), polypropylenegrafted poly(hexamethylene guanidine) (PP-g-PHMG), and $\mathrm{Cu}$ nanoparticles (CuNPs). The effect of CuNP content on the structure, mechanical properties, and antimicrobial properties of the monofilaments was studied. In addition, the antifouling property of fishing nets using the modified polyethylene monofilaments was evaluated. The results showed that CuNPs were dispersed homogeneously in the monofilament matrix as microaggregates. The initial increase in the tensile strength of monofilament was attributed to the mechanical restraint, whereas a decrease in the tensile properties at $1.0 \mathrm{wt} \% \mathrm{CuNP}$ content was due to the predominant effect of a decrease in the total crystallinity. A bacteriostatic test showed that the monofilament had an obvious inhibitory effect on Staphylococcus aureus. Furthermore, coupon test results showed that PP-g-PHMG/PE/CuNP nanocomposite netting had lower weight gain than PP-g-PHMG/PE netting (23.6\% reduction) because of the combined antibacterial effect of both PHMG and CuNP. Therefore, PP-g-PHMG/PE/CuNP monofilament has the potential to produce green and efficient antifouling fishing nets.

\section{Introduction}

Fishing nets of cages, enclosures, and other apparatuses are subjected to biological fouling during the aquaculture process; thus, the organisms involved affect the exchange of water inside and outside, as well as the health of cultured fish [1]. The antifouling methods mainly include the manual cleaning method, the mechanical cleaning method, the antifouling coating method, the metal alloy netting antifouling method, the biological antifouling method, and the intrinsic antifouling method of netting [2]. Manual or physical mechanical removal is time-consuming and laborious [3]. Biological control methods use the competition of organisms to achieve antifouling and only apply to a small number of specific organisms [4]. Antifouling coatings are controversial because they are time-limited and easy to fall off [5]. Metal alloy netting has great antifouling property, but it is heavy and expensive. The intrinsic antifouling method is focused on polymeric molecular structures (micro- and nanostruc- tures [6] or ionic polymer [7]) that show intrinsic antifouling and antimicrobial properties. The antifouling performance of the intrinsic antifouling method is long-lasting. For fishing nets, there is a need for a more environmentally friendly antifouling strategy, able to combine a more efficient and long-lasting antifouling performance.

Polyhexamethylene guanidine hydrochloride (PHMG) is a new cationic bactericidal polymer that has a broad spectrum and is highly efficient, nontoxic, and nonirritating [810]. PHMG is a cationic polymer, and bacteria are electrostatically attracted to the cell membrane, resulting in the rupture of cell membranes, ultimately achieving sterilization [11, 12 ]. Because of its high efficiency and safety, it is widely used in household and hospital disinfection, fabric antibacterial treatment, and other applications [13, 14]. Yang et al. [15] grafted and melted maleic anhydride and PHMG to obtain polypropylene wax (PPW-g-PHMG) to obtain polypropylene with long-term antibacterial properties. Song et al. [16] studied the graft copolymerization of chitosan (CTS) and 
PHMG by amide polycondensation and prepared CTS/PHMG materials with great antibacterial properties. Also, more and more PHMG-modified materials are expected to be used in many fields. Xu et al. reported a novel rapidly deposited polydopamine (PDA) layer with PHMG and found that the PHMG-modified membrane presented excellent antifouling performance [17]. In our previous work [18], PP-gPHMG/PE alloy monofilaments were prepared, and the PP-gPHMG/PE alloy monofilament (with PP-g-PHMG content > $20 \mathrm{wt} \%)$ had excellent antibacterial activity. In addition, we found that the alloy monofilament was typically more active against Staphylococcus aureus than against E. coli.

The significant improvement of nanofiller-modified polymers has attracted the attention of researchers. $\mathrm{Cu}$ nanoparticle (CuNP) and its oxides can release copper ions to seawater, which can prevent the growth of marine organisms. Ananda and Sasikumar [19] obtained some nanomaterials containing silicon, phosphorus, and sulfur. Their antifouling experimental results showed that the nanomaterials had a good inhibitory effect on the adhesion of marine fouling. Molefi et al. [20] melted polyethylene with 0-5\% (volume) CuNP and found that the tensile modulus usually increased with increased CuNP content. When exposed to seawater, CuNP is oxidized to free copper ions, which plays a role in antibacterial and antifouling activity. These nanocomposites have greater application potential in marine antifouling materials.

Marine biofouling is induced by bioslime film, so inhibiting the formation of bioslime film is an important means to solve biofouling [21-23]. In this study, PP-g-PHMG/PE was filled with different CuNP contents, and PP-gPHMG/PE/CuNP monofilaments were prepared by melt blending and spinning. The effects of CuNP content on the structure and properties of PP-g-PHMG/PE/CuNP monofilament were studied. The morphology, crystallization, and segmental dynamics of this monofilament are discussed in detail, and the mechanical and antimicrobial properties are compared with those of bare PP-g-PHMG/PE monofilament. Furthermore, its antifouling performance was also verified by the marine coupon test, providing a reference for its application in fisheries.

\section{Experimental Section}

2.1. Materials. Polyethylene (PE) with an MFI of 0.9 g per 10 min and density of $950 \mathrm{~kg} / \mathrm{m}^{3}$ was supplied by Sinopec Qilu Petrochemical Co., Ltd., China. PP-g-PHMG with a grafting ratio of PHMG of about $10 \%$ was supplied by Shanghai Fuyuan Plastic Technology Co., Ltd., China. CuNP with a size of $50 \mathrm{~nm}$ was provided by Suzhou Canfuo Nanotechnology Co., Ltd., China.

2.2. Preparation of PP-g-PHMG/PE/CUNP Monofilaments. The PP-g-PHMG, PE, and CuNP were blended, extruded, and pelletized by a single screw. Then, the system was treated by melt spinning through a spinning machine with the 0.8 $\mathrm{mm}$ diameter spinneret, and the number of holes is 180 . The temperature of the heating zone of the barrel is $220^{\circ} \mathrm{C}$, $240^{\circ} \mathrm{C}, 260^{\circ} \mathrm{C}, 270^{\circ} \mathrm{C}$, and $270^{\circ} \mathrm{C}$, the length-diameter ratio of screw was $1: 32$, and the screw speed was $20 \mathrm{~m} / \mathrm{min}$. PP-g-PHMG/PE/CuNP monofilaments were prepared by 2-stage draw production. The water bath temperature and the hot air box temperature were $98^{\circ} \mathrm{C}$ and $120^{\circ} \mathrm{C}$, and the draw ratio was 8.5 , under which the monofilaments were continuously spun, winding up the meltspun tow with a winding machine. The diameter of the monofilaments was approximately $0.2 \mathrm{~mm}$, and the line density was approximately 30.0-35.3 tex. The weight ratio of PP-g-PHMG to PE was 20/80, and the content of added CuNP was $0,0.5,1.0$, and $1.5 \mathrm{wt} \%$. Correspondingly, the $\mathrm{PP}-\mathrm{g}-\mathrm{PHMG} / \mathrm{PE} / \mathrm{CuNP}$ monofilament was named as PPg-PHMG/PE, PP-g-PHMG/PE/CuNP-0.5 wt\%, PP-gPHMG/PE/CuNP-1.0 wt\%, and PP-g-PHMG/PE/CuNP$1.5 \mathrm{wt} \%$.

2.3. Characterization. The microstructures of the monofilaments were examined using a scanning electron microscope (SEM) (6360LA, JEOL Ltd., Japan). The sample is fully dried and quenched by liquid nitrogen, the surface of the section is sprayed with gold, and the sample is fixed on the sample table by conductive glue and placed in the electron microscope observation chamber for observation and photographing.

Dynamic mechanical analysis (DMA, 242C, Netzsch Instruments, Germany), tensile mode, used a frequency of $1 \mathrm{~Hz}$ and measured the storage modulus and $\tan \delta$ of composite materials with temperature at a heating rate of $3^{\circ} \mathrm{C} / \mathrm{min}$ from $-180^{\circ} \mathrm{C}$ to $150^{\circ} \mathrm{C}$. Thus, loss factor, $\tan \delta$, is expressed as

$$
\tan \delta=\frac{E^{\prime}}{E^{\prime \prime}}
$$

where $E^{\prime}$ is the storage modulus and $E^{\prime \prime}$ is the loss tangent modulus. $\tan \delta$ is the most fundamental dynamic property of a viscoelastic material.

Differential scanning calorimeter (DSC, 204F1, Netzsch Instruments, Germany), the thermal performance analysis of PP-g-PHMG/PE/CuNP monofilament, was tested with DSC thermal analyzer, protected by nitrogen atmosphere. The sample is heated from $-180^{\circ} \mathrm{C}$ to $150^{\circ} \mathrm{C}$, the heating rate is $10^{\circ} \mathrm{C} / \mathrm{min}$. The degree of crystallinity $\left(X_{c}\right)$ was calculated via the total enthalpy method, according to the following expression:

$$
X_{\mathrm{c}}=\left(\frac{\Delta H_{f}^{\mathrm{obs}}}{\Delta H_{f}^{0}}\right) \times 100 \text {, }
$$

where $\Delta H_{f}{ }^{\text {obs }}$ is the observed heat of fusion values and $\Delta H_{f}{ }^{0}$ is the specific enthalpy of melting for $100 \%$ crystalline polymer. $\Delta H_{f}^{0}$ values of 287 and $209 \mathrm{~J} / \mathrm{g}$ were used for PE and $\mathrm{PP}$, respectively $[24,25]$.

The tensile properties were investigated using an electron tensile tester (4466, Instron Instruments, USA). Test the breaking strength and knotting strength of PP-gPHMG/PE/CuNP monofilament according to the SC/T 5005-2014 specifications under ambient conditions, the 


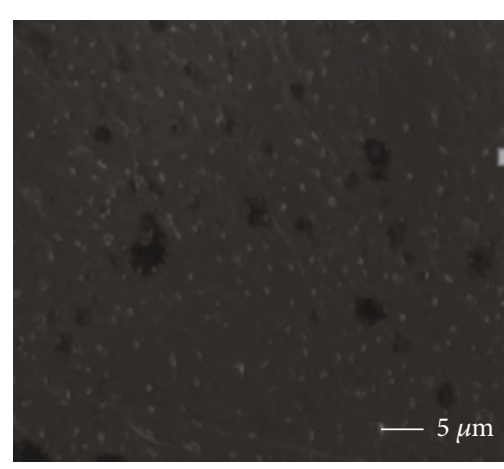

(a)

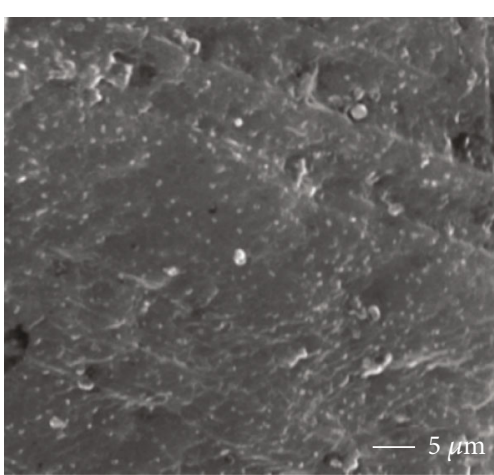

(b)

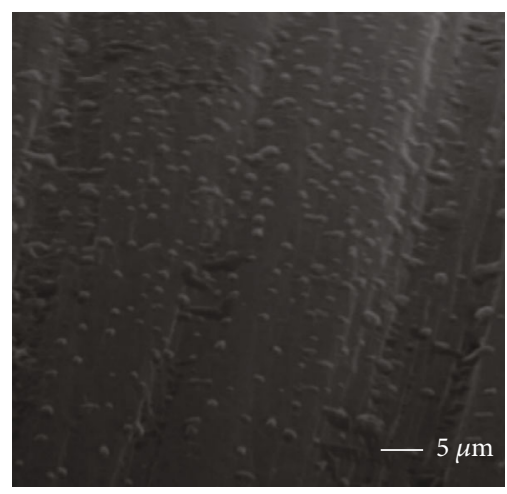

(c)

Figure 1: SEM images of (a) PP-g-PHMG/PE/CuNP-0.5 wt\%, (b) PP-g-PHMG/PE/CuNP-1.0 wt\%, and (c) PP-g-PHMG/PE/CuNP-1.5 wt\% monofilaments. PP-g-PHMG: polypropylene-grafted poly(hexamethylene guanidine); PE: polyethylene; CuNP: copper nanoparticle.

clamping distance is $500 \mathrm{~mm}$, and the tensile speed is 200 $\mathrm{mm} / \mathrm{min}$.

Antimicrobial tests of PP-g-PHMG/PE/CuNP monofilaments with different concentrations were performed using the colony counting method. Staphylococcus aureus (AATCC 6538) was used as representative bacteria. Briefly, UV-sterilized monofilament samples weighing $0.1 \mathrm{~g}$ were orbitally shaken with $500 \mathrm{~mL}$ mother culture broth in an incubator for $24 \mathrm{~h}$ under ambient conditions. Post incubation, serial dilution and vortexing were performed to obtain a concentration of $2.75 \times 10^{6} \mathrm{CFU} / \mathrm{mL}$ in the final solution. Then, $100 \mu \mathrm{L}$ of this solution was swabbed over solidified NB (Nutrient Broth) Petri dishes and kept in an incubator for overnight growth. After culturing for 24 hours, the number of colonies formed on the Petri dish was counted. The following expression to calculate the percentage of reduction of bacterial colonies (antimicrobial activity) was used [26]:

$$
\text { Percentage of antimicrobial activity }=\frac{(A-B) * 100}{A}
$$

where $A$ is the number of colonies formed in control samples and $B$ is the number of colonies formed in PP-g-PHMG/PE/CuNP monofilament.

The antifouling test was conducted by a marine coupon test in the East China Sea $\left(29^{\circ} 55^{\prime} 21.16^{\prime \prime} \mathrm{N}, 122^{\circ} 24^{\prime} 7.87^{\prime \prime} \mathrm{E}\right)$, and the submerged depth was $1 \mathrm{~m}$. The samples were drawn at $0-, 1-$, and 3-month intervals. The retrieved mesh was cleaned by immersing in water to remove sediment and other dirt. Then, it was weighed to assess the accumulation of fouling organisms.

\section{Results and Discussion}

3.1. Microstructure. The dispersion of CuNPs in the matrix was evaluated visually by SEM images, as shown in Figure 1. After adding CuNPs, they were dispersed homogeneously in the monofilament matrix as microaggregates (Figures 1(a)-1(c)), showing an irregular and nonspherical structure. This finding indicates that CuNPs are not completely dissociated and dispersed because of the high melt viscosity during melt spinning. The aggregation of CuNPs is also attributed to the strong van der Waals interaction between the nanoparticles, which is consistent with the results of CuNP/HDPE monofilaments studied by $\mathrm{Yu}$ et al. [27]. When the content was 0.5 and $1.0 \mathrm{wt} \%$, the scale of CuNP aggregates was $400-450 \mathrm{~nm}$ (including 8-9 CuNPs). When the content was $1.5 \mathrm{wt} \%$, the number of CuNP particles was between 12 and 15, forming aggregates with a size of $600 \mathrm{~nm}$.

3.2. Crystallization. DSC is used to analyze the thermal performance of PP-g-PHMG/PE/CuNP monofilaments, and through the DSC analysis curve of PP-g-PHMG/PE/CuNP monofilaments (Figure 2), the crystallinity was calculated 


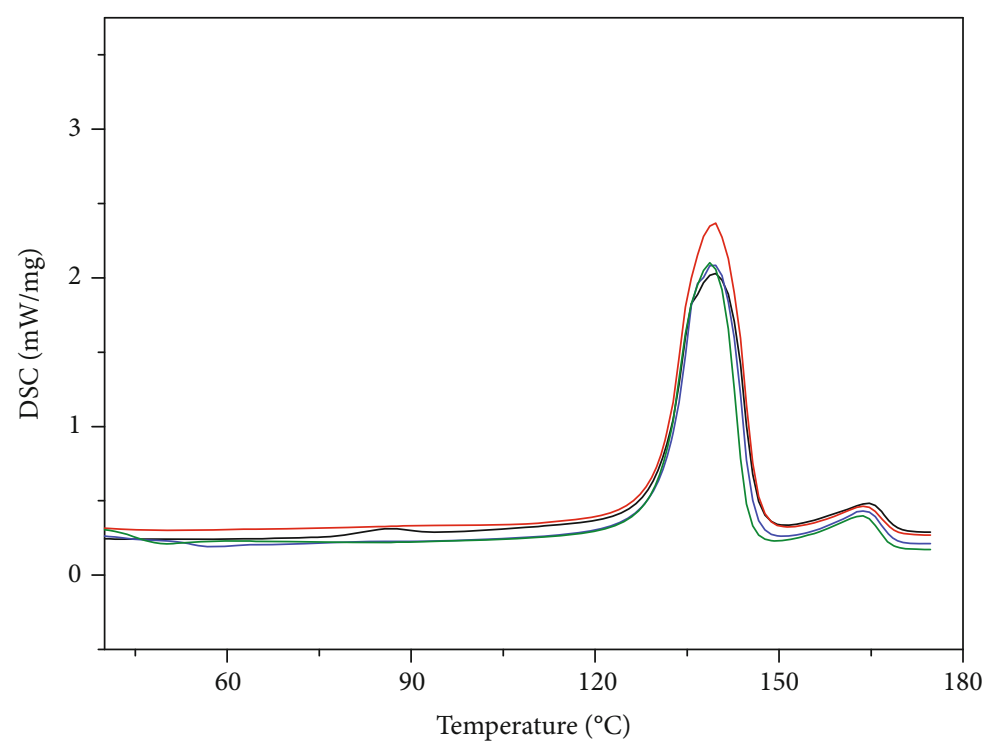

$\begin{array}{ll}\text { PP-g-PHMG/PE } & \text { PP-g-PHMG/PE/CuNP-1.0 wt } \% \\ \text { PP-g-PHMG/PE/CuNP-0.5 wt } \% & \text { PP-g-PHMG/PE/CuNP- } 1.5 \text { wt } \%\end{array}$

(a)

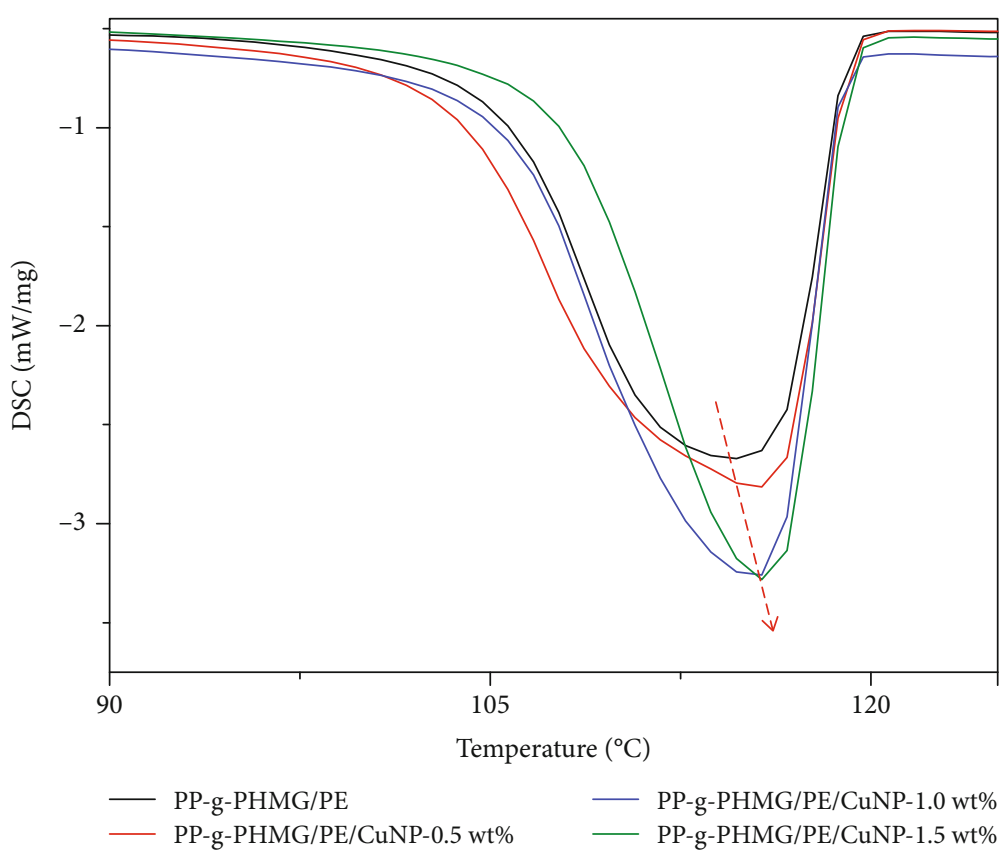

(b)

FIGURE 2: Differential scanning calorimetry (DSC) melting curves of PP-g-PHMG/PE/CuNP monofilaments with different CuNP content under (a) heating and (b) cooling.

TABle 1: $T_{\mathrm{m}}, X_{\mathrm{c}}$, and $T_{\mathrm{c}}$ of PP-g-PHMG/PE/CuNP monofilaments.

\begin{tabular}{lccccc}
\hline Samples & $T_{\mathrm{m}, \mathrm{PE}}\left({ }^{\circ} \mathrm{C}\right)$ & $T_{\mathrm{m}, \mathrm{PP}}\left({ }^{\circ} \mathrm{C}\right)$ & $X_{\mathrm{c}, \mathrm{PE}}$ & $X_{c, \mathrm{PP}}$ & $X_{c, \text { total }}$ \\
\hline PP-g-PHMG/PE & 139.5 & 164.5 & 43.8 & 4.2 & 35.9 \\
PP-g-PHMG/PE/CuNP-0.5 wt\% & 139.4 & 163.8 & 50.3 & 3.5 & 40.9 \\
PP-g-PHMG/PE/CuNP-1.0 wt\% & 139.0 & 163.9 & 44.1 & 4.3 & 36.1 \\
PP-g-PHMG/PE/CuNP-1.5 wt\% & 138.9 & 163.5 & 42.7 & 4.8 & 35.1 \\
\hline
\end{tabular}




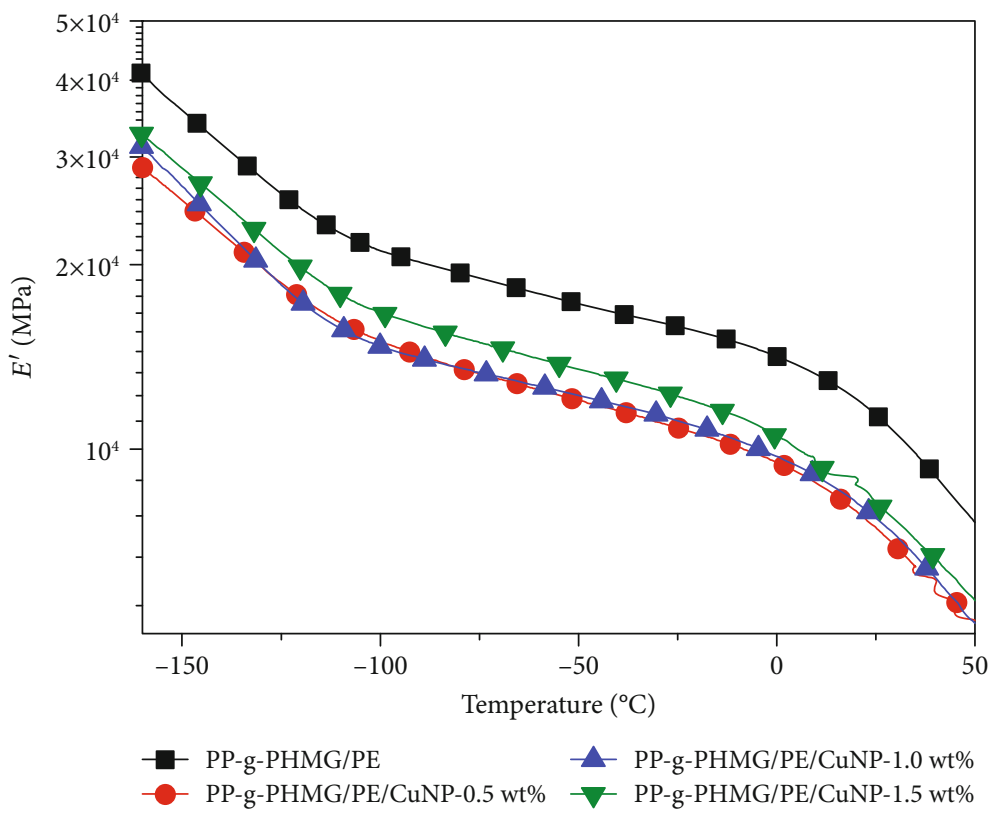

(a)

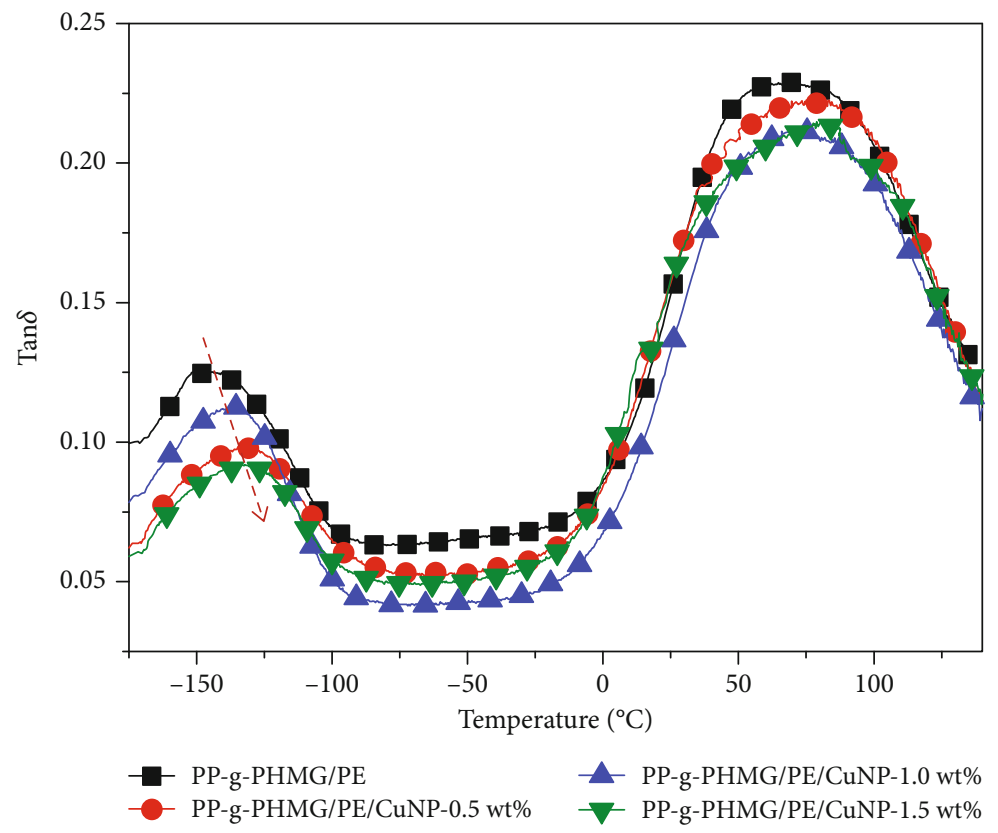

(b)

Figure 3: (a) $E^{\prime}$-T spectrum and (b) tan $\delta$-T spectrum of PP-g-PHMG/PE/CuNP monofilaments with different contents.

based on the melting peak area (Table 1). The total degree of crystallinity (including both phases), $X_{c, \text { total }}$, was calculated as the sum of the degrees of crystallinity of pure components: $X_{\mathrm{c}, \text { total }}=X_{\mathrm{c}, \mathrm{PE}} W_{\mathrm{PE}}+X_{\mathrm{c}, \mathrm{PP}}\left(1-W_{\mathrm{PE}}\right)$, where $X_{\mathrm{c}, \mathrm{PE}}$ and $X_{\mathrm{c}, \mathrm{PP}}$ are the degrees of crystallinity of $\mathrm{PE}$ and $\mathrm{PP}$, respectively, and $W_{\mathrm{PE}}$ is the weight fraction of PE [28].

It can be seen that the composite monofilaments showed two melting endotherms, reflecting two crystalline phases, $\mathrm{PE}$ and $\mathrm{PP}$ phases. The melting point $\left(T_{\mathrm{m}}\right)$ of $\mathrm{PE}$ and $\mathrm{PP}$ is around 139 and $164^{\circ} \mathrm{C}$, respectively. It is noted that the addition of CuNPs had no significant effect on the $T_{\mathrm{m}}$ of the PE and PP matrix. However, compared to pure PP-g-PHMG/PE monofil- aments, the $X_{c, \text { total }}$ of PP-g-PHMG/PE/CuNP monofilaments increased. When the CuNP content was $0.5 \mathrm{wt} \%$, PP-gPHMG/PE/CuNP monofilament had the largest $X_{c, \text { total }}$ of $40.9 \%$, which is $5 \%$ higher than the value for pure PP-gPHMG/PE monofilament. It should be noted that for other systems, low concentrations of nanofillers had also been observed to increase the crystallization rate [29]. Further increment in the CuNP content (1.0 wt\%) caused a noteworthy decrease in the $X_{c, \text { total }}(36.1 \%)$. This was consistent with the reports in literatures [30]. This was mainly due to a dilution effect and hindrance of CuNP particles to polymer molecules that suppressed the molecule rearrangements and crystallization. 


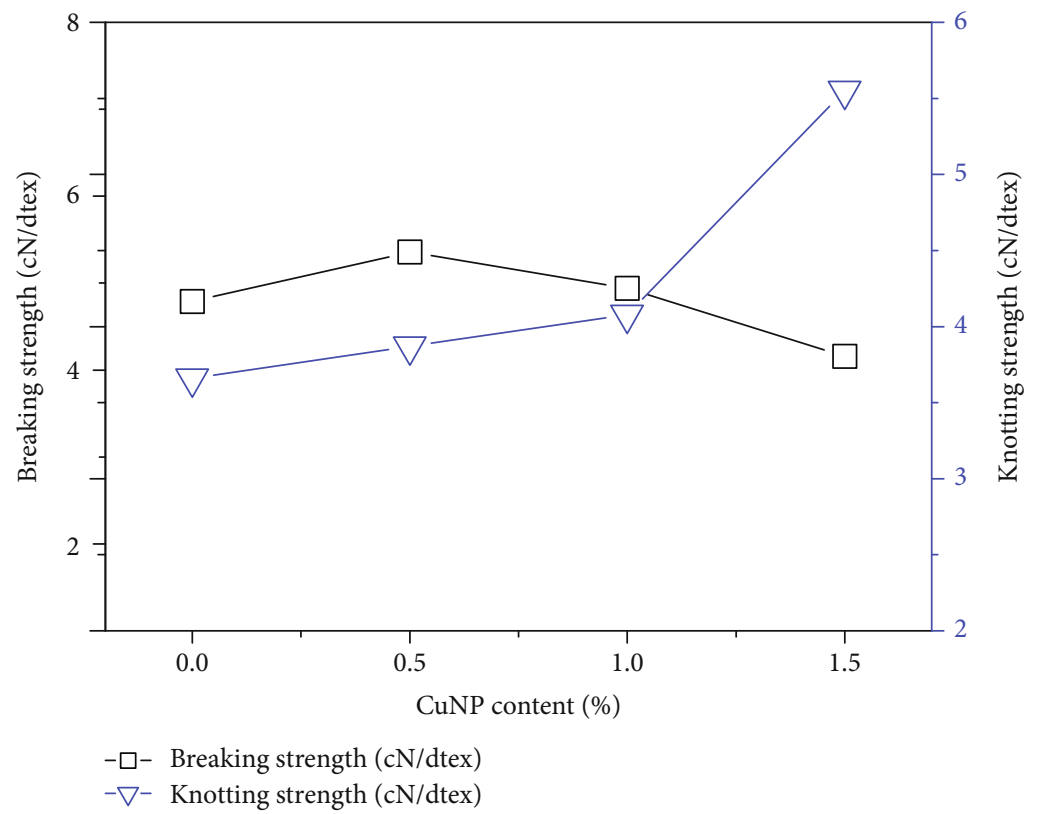

FIGURE 4: Dependence of breaking and knotting strength of PP-g-PHMG/PE/CuNP monofilaments with different CuNP contents.

From Figure 2(b) and Table 1, crystallization temperature $\left(T_{c}\right)$ is found to increase with increased CuNP content. In the crystallization process, the nanofiller serves as nucleation seeds and plays an active role in the heterogeneous nucleation of polymer blends, which promotes crystallization [28]. CuNP serves as nucleation seeds and promotes crystallization, thereby prompting polymer molecular chains to crystal at a high temperature $[31,32]$.

3.3. Viscoelastic Properties. The viscoelastic properties of PPg-PHMG/PE/CuNP monofilament were studied by dynamic mechanical analysis. Figure 3 shows the temperature dependence of its dynamic mechanical properties. The addition of CuNPs reduces the $E^{\prime}$ of the monofilament, which reflects its lower elastic modulus. This means that the CuNP has a plasticization effect on composite monofilament, which is related to the decrease in total crystallinity. However, the $E^{\prime}$ of PP-g$\mathrm{PHMG} / \mathrm{PE} / \mathrm{CuNP}$ monofilament increased with increased CuNP content. This indicates that the stiffness is remarkably enhanced by the increased CuNP content of the monofilament [16]. Ünal et al. also reported a similar phenomenon in nanoclay containing fiber-reinforced hybrid composites [33].

As shown in Figure 3(b), in a wide testing temperature range, $\mathrm{PP}-\mathrm{g}-\mathrm{PHMG} / \mathrm{PE} / \mathrm{CuNP}$ monofilament with different CuNP contents was detected with two relaxation processes. The relaxation transition at low temperature is called $\gamma$ -relaxation, which is associated with the amorphous phase [34]. After the introduction of CuNP, the $T_{\gamma}$ of the monofilament moved to higher temperature compared with PP-g$\mathrm{PHMG} / \mathrm{PE}$, from -147.6 to $-129.2^{\circ} \mathrm{C}$, and the values of $\tan \delta$ for $\gamma$ peak significantly decreased. Usually, the shape and position of the $\tan \delta$ peak are closely related to the molecular structure [35]. This is because the interaction between polymer molecules and CuNP will limit the polymer chain and reduce the activity of the macromolecular chain. The energy required for chain movement becomes higher, which leads to a decreased peak value of $\tan \delta$ and high $T_{\gamma}$. The relaxation transition at a positive temperature is called $\alpha$-relaxation, which corresponds to the motion of restricted chain segments near the crystalline region [36]. The peak value of $\alpha$ decreases with the increase of CuNP content. It may be due to the impediment of thermal motion of polymer due to physical restraints of the CuNP.

3.4. Mechanical Properties. The mechanical properties of PPg-PHMG/PE/CuNP monofilaments are shown in Figure 4. It can be seen that the breaking strength of the monofilaments first increased and then decreased, while knotting strength increased with the increased CuNP content. The breaking strength of the fiber was determined by the microstructure of the fiber [37]. The initial increase in the tensile strength was attributed to the mechanical restraint. With the increase of CuNP content, the breaking strength of the PP-gPHMG/PE/CuNP monofilament showed a downward trend. The mechanical properties of olefin polymer are dependent on its crystallinity. The DSC study found that with the increase of CuNP content, the PP-g-PHMG/PE/CuNP monofilament $X_{c, \text { total }}$ decreased. When the CuNP content was $0.5 \mathrm{wt} \%$, the PP-g-PHMG/PE/CuNP monofilament exhibited better breaking and knotting strength than PP-gPHMG/PE samples. This finding is related to the dispersion state of nanoparticles and the microstructure of the aggregation state. When CuNPs are uniformly dispersed in the PE matrix with good interfacial bonding, significant nanoenhancement and toughening effects are produced, resulting in increased breaking and knotting strength. A further increase in CuNP loading (1.0 wt\%) led to a reduction in breaking strength. When the CuNP content was $1.5 \mathrm{wt} \%$, larger agglomerates brought more defects [25]. Nasibulina 


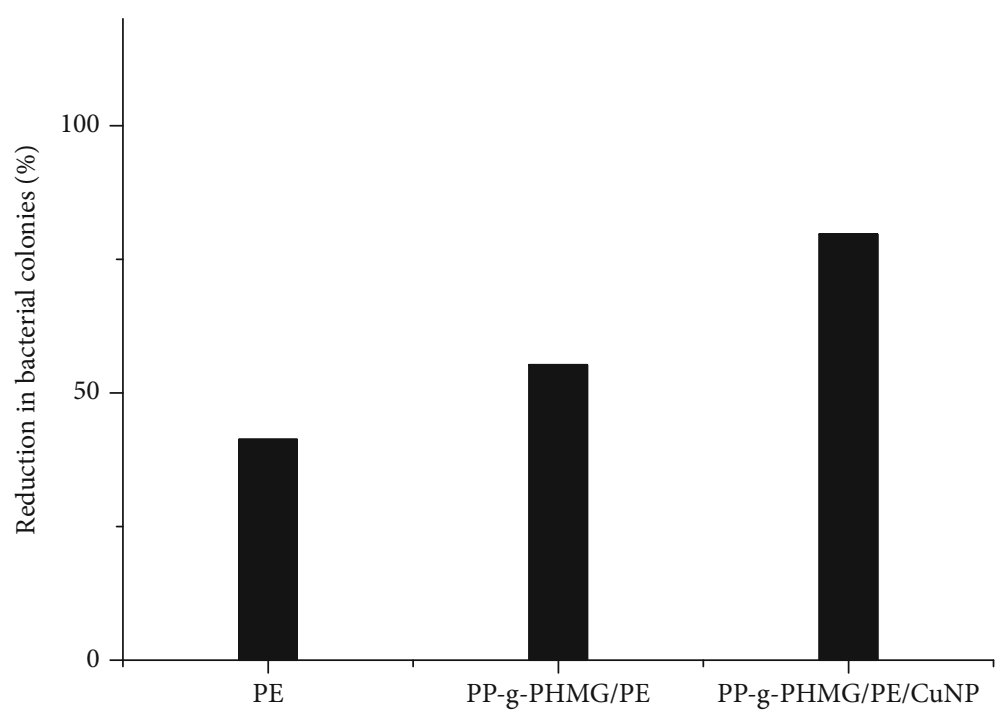

(a)

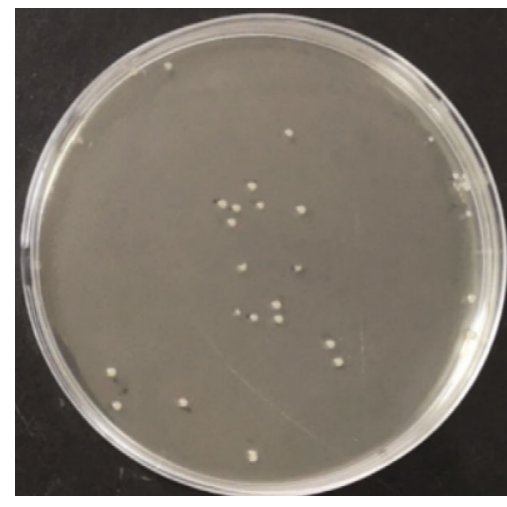

(c)

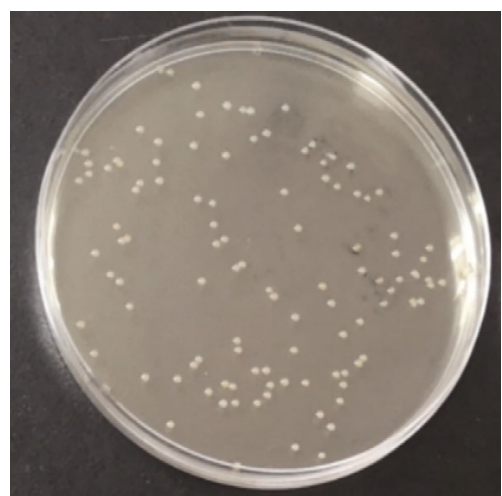

(b)

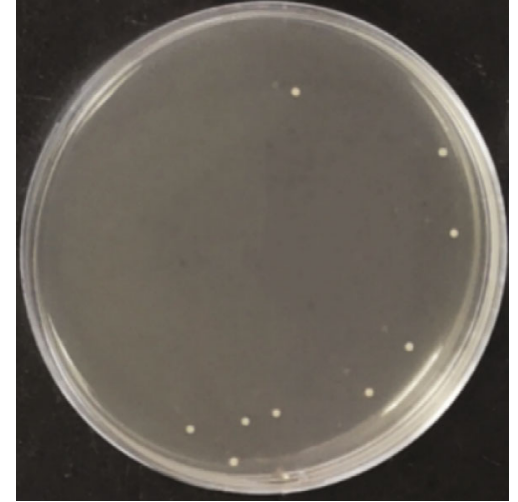

(d)

Figure 5: (a) Antibacterial rate of Staphylococcus aureus after sample culture for $24 \mathrm{~h}$; (b-d) colonies in PE, PP-g-PHMG/PE, and PP-gPHMG/PE/CuNP monofilament culture for $24 \mathrm{~h}$.

et al. [38] found that the use of functionalized nanomaterials could further improve the enhanced mechanical properties.

3.5. Antibacterial Test. CuNP exhibits excellent antimicrobial properties $[39,40]$. In our previous research, we found that PP-g-PHMG/PE had a good inhibitory effect on Staphylococcus aureus [16]. In this study, we chose Staphylococcus aureus as the test bacteria to study the antibacterial properties of PPg-PHMG/PE/CuNP monofilaments. For three samples at a dilution factor of $10^{-5}$, the antibacterial rate of culture cultivated for 24 hours was measured, shown in Figure 5(a). Figures 5(b)-5(d) show photographs of the colonies of the three samples cultured for $24 \mathrm{~h}$. The antibacterial effect of PP-g-PHMG/PE/CuNP monofilament was significantly better than that of the other two. After calculation, compared to PP-g-PHMG/PE monofilament, the reduction in bacterial colonies of PP-g-PHMG/PE/CuNP monofilament increased by $44.2 \%$. This attributes to the bactericidal property of CuNP in the composite monofilament. The metal cations released from the unstable CuNP can form pits on the cell wall, causing cytoplasmic fluid leakage. Once inside the cell, the metal cations will generate excessive amounts of highly reactive free radicals called reactive oxygen species (ROS). These free radicals are by-products of the Fenton reaction. ROS attack the double-helix structure of bacterial DNA, which will accelerate cell apoptosis [41].

3.6. The Antifouling Effect of Fishing Netting. PE, PP-gPHMG/PE, and PP-g-PHMG/PE/CuNP-1.5 wt\% monofilaments were twisted into $4 \times 3$ netting twine to prepare knotted netting with a mesh length of $40 \mathrm{~mm}$. The coupon test was used to evaluate antifouling performance from August to October 2020 in a shallow area of the East China Sea.

As shown in Table 2, one month later, the three nets had a small amount of attachment, but the attached area on the PE netting was larger than the other two. Three months later, the PE netting formed lots of attached organisms. After the introduction of PP-g-PHMG and CuNP, the PP-gPHMG/PE and PP-g-PHMG/PE/CuNP netting eventually formed less deposits. Among them, the PP-g-PHMG/PE/CuNP nanocomposite netting had fewer barnacles attached to the surface than the PP-g-PHMG/PE netting. In order to make quantitative analysis of attached organisms, the types of netting from the same area were cropped and weighed. 
TABLE 2: Coupon test results of PE, PP-g-PHMG/PE, and PP-g-PHMG/PE/CuNP fishing nets.

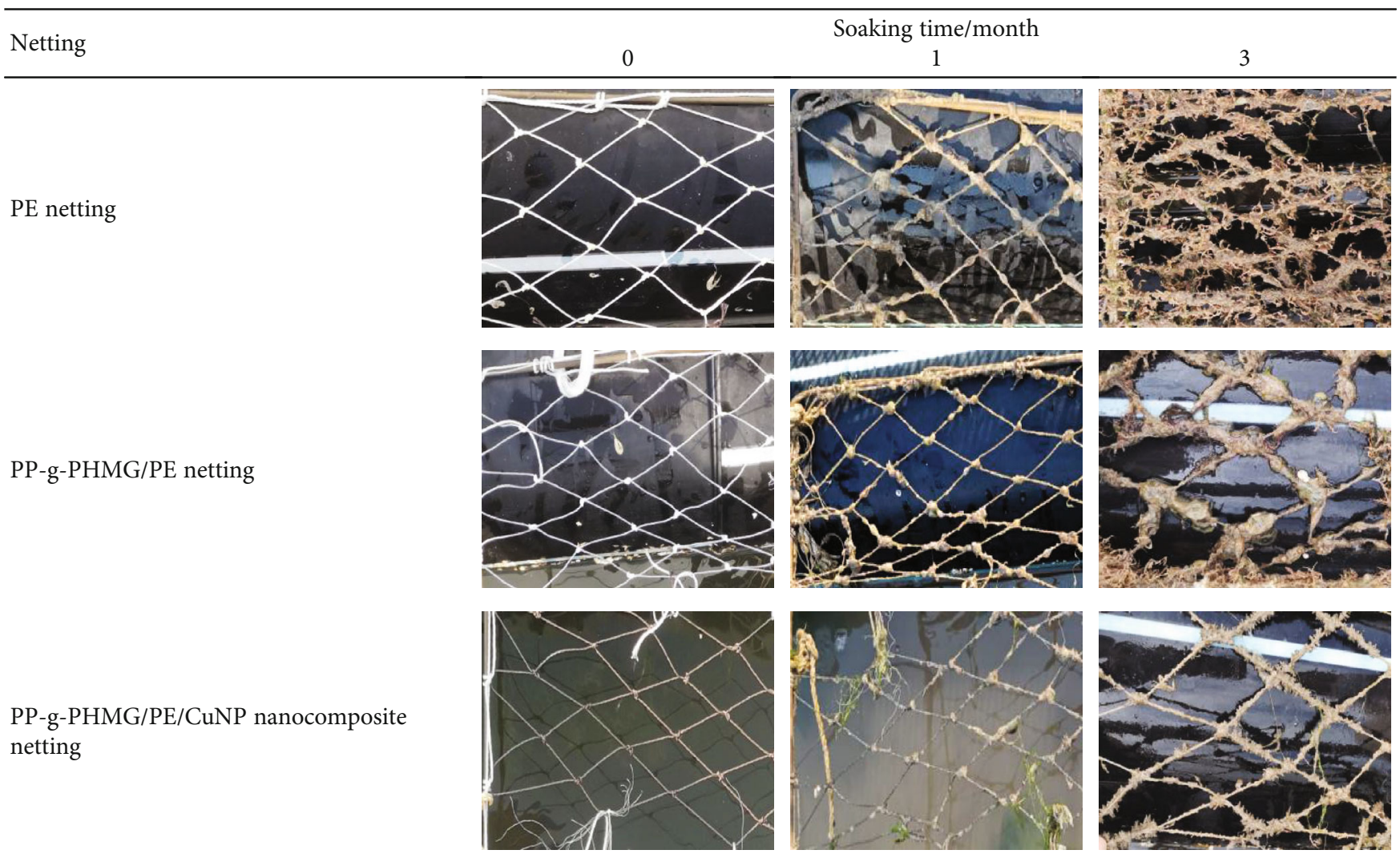

The results show that the PP-g-PHMG/PE/CuNP nanocomposite netting showed lower weight gain (23.6\% reduction) than the PP-g-PHMG/PE netting. Hence, the PP-gPHMG/PE/CuNP nanocomposite has a certain repellent effect on attaching organisms. The result occurs for two reasons: (1) the bacterial cell wall carries a net negative charge due to the presence of phospholipids, so there is an electrostatic attraction between the cation and the cell wall. PHMG is a cationic polymer, and bacteria are electrostatically attracted to the cell membrane, resulting in failure of the cell membrane, and (2) the metal cations released from the unstable CuNP can form pits on the cell wall, causing cytoplasmic fluid leakage. The adhesion of fouling organisms starts from the adhesion of bacteria and other prokaryotic microorganisms and the formation of biofilms, so the PP-g$\mathrm{PHMG} / \mathrm{PE} / \mathrm{CuNP}$ with great antibacterial effect is a potential fishing net material to decrease biofouling.

\section{Conclusion}

The effect of CuNP content on the structure, mechanical properties, and antimicrobial and antifouling properties of PP-g-PHMG/PE/CuNP monofilaments was studied.

CuNPs were dispersed in the PP-g-PHMG/PE/CuNP monofilament matrix as microaggregates, showing an irregular and nonspherical structure. After adding CuNPs, the total crystallinity of the monofilament increased. The $\alpha$-relaxation associated with the crystalline region of the polymer matrix became weaker with the increased CuNP content. The break- ing and knotting strength of PP-g-PHMG/PE/CuNP monofilament with low CuNP content was superior to those of PP-g-PHMG/PE monofilament. When CuNP content was increased to $1.0 \mathrm{wt} \%$, the mechanical properties decreased, as a result of the aggregation of CuNPs.

In addition, the antibacterial activity of PP-gPHMG/PE/CuNP monofilament showed an obvious advantage. We found that the antibacterial effect of the CuNP monofilament on Staphylococcus aureus was 38.36\% higher than that of PE monofilament. The immobilization of the biocide (PHMG and CuNP) into the matrix will potentially lead to a more efficient, long-lasting antifouling performance in water-immersed structures. Moreover, the results of the antifouling test showed that the weight gain of the PP-gPHMG/PE/CuNP nanocomposite netting was lower than that of the PP-g-PHMG/PE netting (23.6\% reduction) and PE netting.

\section{Data Availability}

The data used to support the findings of this study are included within the article.

\section{Conflicts of Interest}

The authors declare that they have no conflicts of interest. 


\section{Acknowledgments}

This study is supported by the National Natural Science Foundation of China (31972844 and 31872611), Ministry of Industry and Information Technology high tech ship research project (engineering development of semisubmersible agriculture equipment), National Key R\&D Plan (2020YFD0900803), China Agriculture Research System (CARS-50), Key Program of Marine Economy Development (Six Marine Industries) Special Foundation of Department of Natural Resources of Guangdong Province (GDNRC [2020]016), and the Fund for Shanxi "1331 Project".

\section{References}

[1] X. Ma, S. J. Liu, X. H. Sui, X. Q. Zhou, and C. H. Xia, "Preparation and assessment of antifouling coatings for polyethylene based fishing net," Paint \& Coatings Industry, vol. 46, pp. 33$38,2016$.

[2] J. G. Shi, Agriculture Technology of Deep-Sea Ecological Enclosure, Ocean Press, 2019.

[3] L. H. GUO, "Mechanical removal of cage attachment biological defects," Scientific Fish Farming, vol. 4, p. 15, 1994.

[4] M. Pagliaro, R. Ciriminna, and F. V. Bright, "Ecofriendly antifouling marine coatings," Acs Sustainable Chemistry and Engineering, vol. 3, no. 4, pp. 559-565, 2015.

[5] B. Ashok, N. Hariram, S. Siengchin, and A. V. Rajulu, "Modification of tamarind fruit shell powder with in situ generated copper nanoparticles by single step hydrothermal method," Journal of Bioresources and Bioproducts, vol. 5, no. 3, pp. 180-185, 2020.

[6] M. Milazzo, G. Gallone, E. Marcello et al., "Biodegradable polymeric micro/nano-structures with intrinsic antifouling/antimicrobial properties: relevance in damaged skin and other biomedical applications," Journal of Functional Biomaterials, vol. 11, no. 3, p. 60, 2020.

[7] X. Zhang, L. Liu, W. Peng et al., "Phosphonate/zwitterionic/cationic terpolymers as high-efficiency bactericidal and antifouling coatings for metallic substrates," Journal of Materials Chemistry B, vol. 9, no. 20, pp. 4169-4177, 2021.

[8] C. Wei, D. F. Wei, A. Zheng, and Y. Guan, "Surface enrichment and nonleaching antimicrobial performance of polypropylene grafted poly(hexamethylene guanidine) (PP-g-PHMG) in poly(ethylene terephthalate)/PP-g-PHMG," European Polymer Journal, vol. 118, pp. 231-238, 2019.

[9] S. R. Suprakas and O. Masami, "Polymer/layered silicate nanocomposites: a review from preparation to processing," Progress in Polymer Science, vol. 28, pp. 1539-1641, 2003.

[10] J. Nillola, X. Liu, Y. Li et al., "Surface modification of thin film composite RO membrane for enhanced anti-biofouling performance," Journal of Membrane Science, vol. 444, pp. 192200, 2013.

[11] M. L. Wllace, "Testing the efficacy of polyhexamethylene biguanide as an antimicrobial treatment for cotton fabric," AATCC Review, vol. 1, no. 11, pp. 18-20, 2001.

[12] X. F. Sun, Y. Q. Gao, S. Zhao, W. Zhang, Z. Wang, and X. L. Wang, "Ultrafiltration membrane with antibacterial and antifouling properties by grafting guanidine based polymer," CIESC Journal, vol. 69, no. 11, pp. 4869-4878, 2018.

[13] Z. X. Zhou, D. F. Wei, Y. Guan, A. N. Zheng, and J. J. Zhong, "Damage of Escherichia coli membrane by bactericidal agent polyhexamethylene guanidine hydrochloride: micrographic evidences," Journal of Applied Microbiology, vol. 108, no. 3, pp. 898-907, 2010.

[14] M. Walczak, A. Richert, and A. Burkowska-But, "The effect of polyhexamethylene guanidine hydrochloride (PHMG) derivatives introduced into polylactide (PLA) on the activity of bacterial enzymes," Journal of Industrial Microbiology \& Biotechnology, vol. 41, no. 11, pp. 1719-1724, 2014.

[15] J. Yang, H. L. Cheng, D. F. Wei, Y. Guan, and A. N. Zhang, "Modification of polypropylene wax by bonding polyhexamethylene guanidine and its antimicrobial activity," Journal of Functional Polymers, vol. 25, no. 4, pp. 364-368, 2012.

[16] Y. Song, L. Yao, X. Wang, Z. Gu, and S. Wu, "Preparation and characterization of antibacterial agent PHMG modified chitosan," China Adhesives, vol. 24, no. 10, pp. 19-22, 2015.

[17] X. C. Xu, C. Q. Zhao, G. Q. Zhang, and F. L. Yang, "Rapidly self-assembled polydopamine coating membranes with polyhexamethylene guanidine: formation, characterization and antifouling evaluation," Colloids and Surfaces A: Physicochemical and Engineering Aspects, vol. 512, pp. 41-50, 2017.

[18] Y. Wang, W. W. Yu, A. Y. Shu, J. G. Shi, and M. Zhang, "Polypropylene-grafted poly(hexamethylene guanidine)/modified polyethylene monofilament and its antimicrobial performance," International Journal of Polymer Science, vol. 2020, no. 2, Article ID 6416230, pp. 1-8, 2020.

[19] S. K. Ananda and A. Sasikumar, "Studies on novel silicone/phosphorus/sulphur containing nano-hybrid epoxy anticorrosive and antifouling coatings," Progress in Organic Coatings, vol. 68, no. 3, pp. 189-200, 2010.

[20] J. A. Molefi, A. S. Luyt, and I. Krupa, "Comparison of the influence of copper micro- and nano-particles on the mechanical properties of polyethylene/copper composites," Journal of Materials Science, vol. 45, no. 1, pp. 82-88, 2010.

[21] Z. Guo, J. Xue, T. Liu, X. Song, Y. Shen, and H. Wu, “Antibacterial mechanisms of silica/polydopamine/silver nanoparticles against gram positive and gram negative bacteria," Micro \& Nano Letters, vol. 9, no. 3, pp. 210-214, 2014.

[22] K. M. Peng, W. Ding, W. P. Tu, J. Hu, C. Liu, and J. Yang, "Construction of guanidinium-rich polymers and their applications," Acta Chimica Sinica, vol. 74, no. 9, pp. 713-725, 2016.

[23] J. G. Shi, W. W. Yu, K. Zhao et al., "Progress on research of antifouling technology of offshore cage netting," Journal of Fisheries of China, vol. 45, no. 3, pp. 474-487, 2020.

[24] J.-H. Lin, Y.-J. Pan, C.-F. Liu et al., "Preparation and compatibility evaluation of polypropylene/high density polyethylene polyblends," Materials, vol. 8, no. 12, pp. 8850-8859, 2015.

[25] Y. H. Shi and Q. Dou, "Effect of $\beta$-nucleating agent on the crystallization, mechanical properties, and heat resistance of injection-molded isotactic polypropylene," Journal of Macromolecular Science, Part B, vol. 52, no. 3, pp. 476-488, 2013.

[26] A. Roy, M. Joshi, and B. S. Butola, “Antimicrobial performance of polyethylene nanocomposite monofilaments reinforced with metal nanoparticles decorated montmorillonite," Colloids and Surfaces B: Biointerfaces, vol. 178, pp. 87-93, 2019.

[27] W. W. Yu, Y. L. Liu, and L. Wang, "Cu nanoparticle-modified high-density polyethylene monofilament and its antifouling performance on fishing netting," International Journal of Polymer Science, vol. 2019, Article ID 7250348, 10 pages, 2019. 
[28] N. Avramova, "Amorphous poly(ethylene terephthalate)/poly(butylene terephthalate) blends: miscibility and properties," Polymer, vol. 36, no. 4, pp. 801-808, 1995.

[29] D. Homminga, B. Goderis, I. Dolbnya, and G. Groeninckx, "Crystallization behavior of polymer/montmorillonite nanocomposites. Part II. Intercalated poly( $\varepsilon$-caprolactone $) /$ montmorillonite nanocomposites," Polymer, vol. 47, no. 5, pp. 1620-1629, 2006.

[30] Z. T. Yao, T. Chen, H. Y. Li, M. S. Xia, Y. Ye, and H. Zheng, "Mechanical and thermal properties of polypropylene (PP) composites filled with modified shell waste," Journal of Hazardous Materials, vol. 262, pp. 212-217, 2013.

[31] M. E. Babiker and Y. Muhuo, "The effects of loading of montmorillonite (MMT) on the properties of the ultra-high molecular weight polyethylene (UHMWPE/organo-MMT) nanocomposite sheets prepared by gel and pressure induced flow (PIF) processing," Arabian Journal for Science and Engineering, vol. 38, no. 3, pp. 479-490, 2013.

[32] X. Q. Zhang, Y. B. Tan, Y. H. Li, and G. Zhang, "Effect of OMMT on microstructure, crystallisation and rheological behaviour of UHMWPE/PP nanocomposites under elongation flow," Plastics, Rubber and Composites, vol. 47, no. 7, pp. 315-323, 2018.

[33] H. Y. Ünal, G. Öner, and Y. Pekbey, "Comparison of the experimental mechanical properties and DMA measurement of nanoclay hybrid composites," European Mechanical Science, vol. 2, no. 1, pp. 31-36, 2017.

[34] K. Jordens, G. L. Wilkes, J. Janzen, D. C. Rohlfing, and M. B. Welch, "The influence of molecular weight and thermal history on the thermal, rheological, and mechanical properties of metallocene-catalyzed linear polyethylenes," Polymer, vol. 41, no. 19, pp. 7175-7192, 2000.

[35] R. Popli, M. Glotin, L. Mandelkern, and R. S. Benson, "Dynamic mechanical studies of $\alpha$ and $\beta$ relaxations of polyethylenes," Journal of Polymer Science Polymer Physics Edition, vol. 22, no. 3, pp. 407-448, 1984.

[36] K. Sever, I. H. Tavman, Y. Seki, A. Turgut, M. Omastova, and I. Ozdemir, "Electrical and mechanical properties of expanded graphite/high density polyethylene nanocomposites," Composites Part B: Engineering, vol. 53, pp. 226-233, 2013.

[37] X. Y. Chen and Y. P. Liu, "Effect of grafting treatment on the crystalline and physical properties of bamboo fibre," Shanghai Textile Science \& Technology, vol. 35, no. 9, pp. 10-12, 2007.

[38] L. I. Nasibulina, I. V. Anoshkin, A. G. Nasibulin, A. Cwirzen, V. Penttala, and E. I. Kauppinen, "Effect of carbon nanotube aqueous dispersion quality on mechanical properties of cement composite," Journal of Nanomaterials, vol. 2012, Article ID 169262, 6 pages, 2012.

[39] S. Li, D. Wei, Y. Guan, and A. Zheng, "Preparation and characterization of a permanently antimicrobial polymeric material by covalent bonding," European Polymer Journal, vol. 51, pp. 120-129, 2014.

[40] Y. Assem and A. I. Khalaf, "Preparation and characterization of polymeric nanofibers by electrospinning as po\tential antibacterial materials," Colloid and Surface Science, vol. 2, pp. 66-75, 2017.

[41] M. R. Spitzmiller, Interactions of Copper Nanoparticles with the Microalgae, Chlamydomonas Reinhardtii, 2012. 